\title{
Study of Central Venous Cut-Down Versus Percutaneous Central Venous Catheterization in Neonates and Infants \\ ${ }^{1}$ Mohamed Mohamed Ellithy, ${ }^{2}$ Ashraf Abdelhameed Abdelmonem, ${ }^{1}$ Ahmed Abdelghaffar Hamed Helal, ${ }^{1}$ Ibrahim Abu Zeid Ibrahim El Gohary \\ ${ }^{1}$ Department of Pediatric Surgery, ${ }^{2}$ Department of General Surgery, \\ Faculty of Medicine -Al Azhar University \\ Corresponding author: Ibrahim Abu Zeid Ibrahim El Gohary, Mobile: 01144636947
}

\begin{abstract}
Background: Several more access routes of have been described. Umbilical artery catheters (UACs), and umbilical vein catheters (UVCs). CVCs can be placed in numerous sites, including the internal jugular vein (IJV), the subclavian vein, the femoral vein, peripheral veins leading to central access, and other surgical access sites.

Objective: To compare central venous cut-down catheterization versus percutaneous central venous catheterization in neonates and infants regarding technical feasibility, indications, operative time and complications.

Patients and Methods: This is a prospective study on two different methods for central line insertion in neonates and infants. This study was done at Al-Azhar University Hospitals, in Pediatric Surgery Department, Neonatal Intensive Care Unit and Pediatric Intensive Care Unit. One hundred patients who required central venous catheterization were included in this study, during period between December 2018 and October 2019.

Results: The present study reported that the ultrasound guided CVC was more useful in placement of CVC in infants and neonates, and decreasing complications. The ultrasound technique not only clarified the relative position of the vein and its surrounding structures but also helped in identifying the course of the central vein and its caliber and provide confidence to the operator

Conclusion: The present study reported that the ultrasound guided CVC was more useful in placement of CVC in infants and neonates, and decreasing complications. Cost is one of the limiting factors in the availability of ultrasound device in many clinical applications.
\end{abstract}

Keywords: Central Venous Cut-Down, Percutaneous Central Venous Catheterization, Neonates and Infants.

\section{INTRODUCTION}

The need for vascular access in the pediatric patient is frequent (1). However, placement of a peripheral line may not be feasible or appropriate especially in neonates and infants. The options available to clinicians have increased over the years.

The history of cannulation of a central venous structure can be traced back to 1929 , when Forssmann described advancing a plastic tube near the heart by puncturing his own arm ${ }^{(2)}$. In the 1950s, Aubaniac used the subclavian vein to insert a central venous catheter (CVC) ${ }^{(3)}$.

Since then, several more access routes have been described. Umbilical artery catheters (UACs), and umbilical vein catheters (UVCs). (CVCs) can be placed in numerous sites, including the internal jugular vein (IJV), the subclavian vein, the femoral vein, peripheral veins leading to central access, and other surgical access sites ${ }^{(4)}$.

Recently, mean good device is likely to focus on the material used in a central venous catheter (CVCs), as well as on means of preventing infection and occlusion. Ultrasonography is becoming an increasingly useful adjunct in the placement of percutaneous central lines (4). Pediatric and emergency medicine training programs benefit from teaching and using ultrasonography for central line placement ${ }^{(5)}$.
A central venous catheter (CVC), also known as a central line, central venous line, or central venous access catheter, is a catheter placed into a large vein. Catheters can be placed in veins in the neck (internal jugular vein), chest (subclavian vein or axillary vein), groin (femoral vein), or through veins in the arms (also known as a PICC line, or peripherally inserted central catheters). It is used to administer medication or fluids that are unable to be taken by mouth or would harm a smaller peripheral vein, obtain blood tests (specifically the "central venous oxygen saturation"), and measure central venous pressure (6).

\section{AIM OF THE STUDY}

The aim of this study is to compare central venous cut-down catheterization versus percutaneous central venous catheterization in neonates and infants regarding technical feasibility, indications, operative time and complications.

\section{PATIENTS AND METHODS}

This is a prospective study on two different methods for central line insertion in neonates and infants. This study was done at Al-Azhar University Hospitals, in Pediatric Surgery Department, Neonatal Intensive Care Unit and Pediatric Intensive Care Unit. One hundred 
patients who required central venous catheterization were included in this study, from period between December 2018 and October 2019.

Inclusion criteria: All neonates and infants who needed central venous line insertion. All type of central venous access in internal jugular vein, subclavian vein.

Exclusion criteria: Neonates and Infants with coagulopathy. Patients who had a history of previous neck surgery, head and neck mass, distorted anatomy, skeletal deformity, infection or scarring in the site of insertion, or children with pneumothorax or hemothorax.

Patients were divided into two equal groups: Group (A): 50 patients submitted to central venous catheterization by surgical cutdown technique. Group (B): 50 patients submitted to central venous catheterization by ultrasound guided percutaneous technique.

All patients were subjected to:

1-Complete history taken as recorded in patients files stressing upon: Age, sex, cardiorespiratory status, bleeding tendency, indication for CVC insertion whether it is failure of any other peripheral lines or for the need of special drugs or for the assessment of central venous pressure, time of insertion, site of insertion, number of trials, number of CVCs inserted to the patient during his stay in hospital.

2. Physical examination: All patients were assessed for routine cardiac, chest, abdomen, neurological examination, uncorrected bleeding diathesis, skin infection over the puncture site, pneumothorax or hemothorax or the presence of only one functioning lung, skeletal deformity or scarring.

3. Pre-procedures preparation: The following items was considered: The procedure explained to the parents. Oxygen through the nasal catheter was given. ECG monitor and pulse oximetry were connected to the patient. The procedure was carried out under local anesthesia with conscious sedation in all infants and neonates. General anesthesia was used only for babies in the operating room.

Investigations: Pre-insertion (Bleeding time, clotting time, prothrombin time, partial thromboplastin time, international normalized ratio and echocardiography). Any bleeding disorders were corrected as necessary pre-insertion. Postinsertion (Complete blood count, blood culture, Creactive protein and chest $\mathrm{X}$-ray).

Technique of central venous catheterization: All CVC insertion were performed under complete aseptic preparation. Standard monitoring (electrocardiogram, and pulse oximeter) were applied to all patients through the procedure.

In group (A): CVC was inserted in the internal jugular vein using venous cut down technique.

In group (B): CVC was inserted in internal jugular vein, subclavian vein using percutaneous central venous catheterization technique under sonar guide.

CVC diameter: Appropriate size of the catheter was selected $(3,4$, or 5 Fr triple-lumen catheter), default CVC external diameters per patient weight were decided upon at the beginning of the study and remained unchanged: $<3 \mathrm{~kg}: 3$ French, 3-10 kg: 4 Fr, 10-25 kg: 5 Fr.

\section{Group (A) Surgical cut down technique}

All patients were positioned in the Trendelenburg $\left(20-30^{\circ}\right)$ position (head down, to increase the size of the vein and prevent air embolism) with head turned slightly toward the other side and stabilized with folded towels. The length of the catheter insertion was estimated according to patient height by using the following equation: Length of catheter insertion $(\mathrm{cm})=$ (height in $\mathrm{cm} / 10$ )-1 for patients less than or equal $100 \mathrm{~cm}$ in height, and (height in $\mathrm{cm} / 10$ )- 2 for patients more than $100 \mathrm{~cm}$ in height.

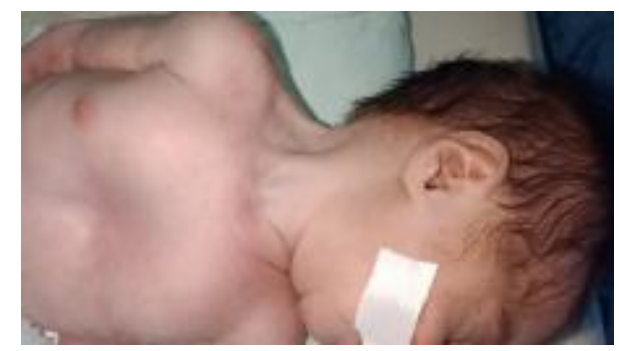

Fig. (1)

Anatomical

landmarks (sternocleidomastoid muscles, sternal notch, and clavicle) were assessed and marked. Small transverse incision (1 to $2 \mathrm{~cm}$ ) was done above the clavicle by 1 to $2 \mathrm{~cm}$. Then two head of the sternomastoid muscle was splitted to visualize the internal jugular vein.

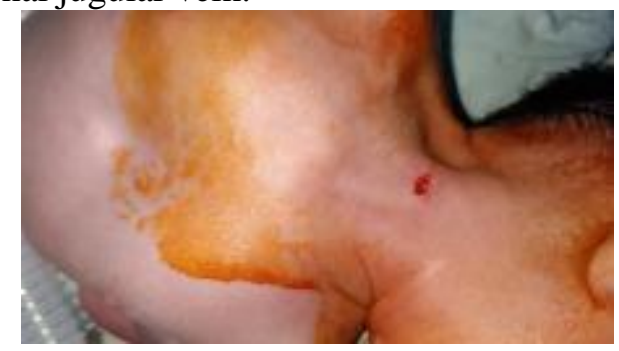

Fig. (2)

Curved mosquito was passed behind the IJV vein, then proximal and distal ligatures of 4-0 absorbable suture was applied loosely around the 
vein (attention was taken not to twist the vein as the suture was advanced).

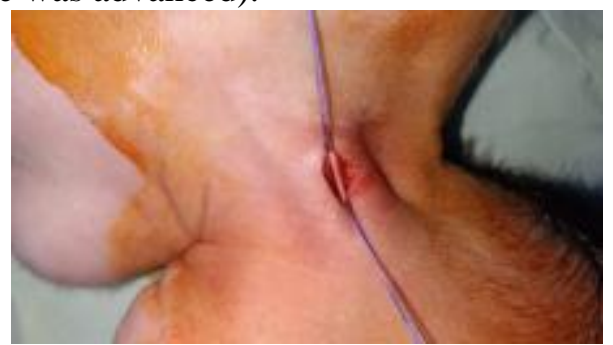

Fig. (3)

Subcutaneous tract was created far enough from site of incision. In the anterior chest wall, the tunnel was created medial to the nipple (far from the breast bud). The end of the catheter was introduced through the tunnel, and the catheter was guided gently through the subcutaneous tract. The catheter system was filled with heparinized flush solution. Transverse venotomy was performed (to avoid ligation of the vein, purse-string suture of 60 polypropylene was placed in the vessel wall around the venotomy incision). Catheter was grasped gently with blunt non-toothed forceps and introduced inside the vein through the venotomy incision.

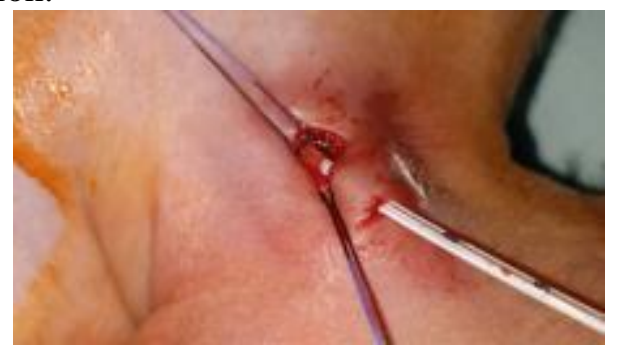

Fig. (4)

Wound was closed with 4-0 absorbable suture, care was taken not to penetrate the catheter. Fixation and sterile dressing was applied.

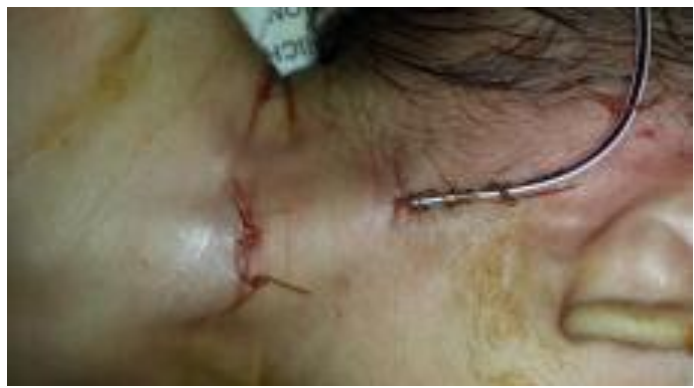

Fig. (5)

Group (B) Ultrasound guided percutaneous technique

a-For internal jugular vein catheterization: The patient was placed in the 15- to 20-degree Trendelenburg position. A towel roll was placed under the shoulders running laterally so that the patient's neck was safely hyperextended as long as such a position was clinically safe. The head was turned away from the site of line placement. The linear Ultrasound probe was used. After the sterile field was prepped, gel was applied to the probe and placed within a sterile cover. The ultrasound probe was oriented transverse to the neck veins near the carotid pulse. The IJV was identified, which was usually lateral and anterior to the carotid artery. With downward pressure from the probe, the IJV was usually compressible while the carotid artery was pulsatile. Once the IJV was identified, the probe was centred over the vessel (Fig. 4).

The needle was inserted into the skin at a 30to 45-degree angle at the midline of the probe near where it contacts the skin. The sterile cover of the probe was not punctured. With the probe visualizing the vessel transversely, slowly the needle was advanced and by sliding the probe toward ipsilateral nipple. The ultrasound probe was advanced until the needle punctures the vessel wall. The Seldinger technique was proceeded after venepuncture. When there was free flowing dark venous blood return, a guide wire was inserted through the needle into the vein. The needle was removed and the guide wire was hold firmly. The skin entry site was enlarged with a small dilator. The ultrasound was placed parallel to the vessel to view the guide wire, if desired (Fig. 1).

The right side IJV was preferable because of its straight course for the catheter to the right atrium, absence of thoracic duct, and lower pleural dome. Chest radiograph was obtained to confirm proper catheter placement and rule out pneumothorax. A sterile dressing was applied over the site.

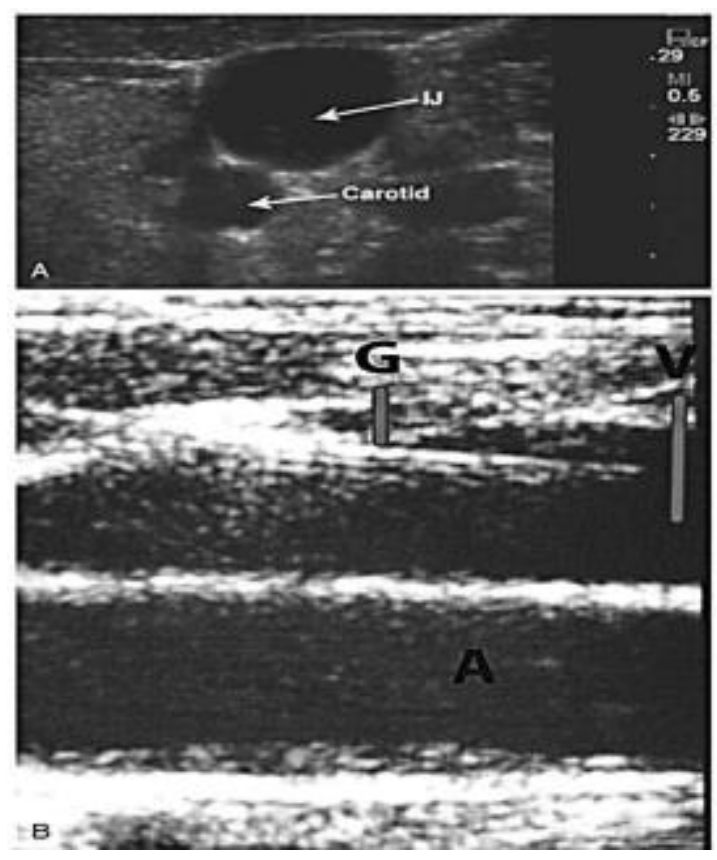

Figure (6): Ultrasound view of IJV

A- Ultrasound transverse view of IJV and carotid artery. In this short axis view, the internal jugular vein IJV is seen anterior and lateral to the carotid artery. The artery is often smaller and 
pulsatile while the IJV is usually larger and compressible. Just anterior to the IJV is the sternocleidomastoid muscle. B- Ultrasound longitudinal view of IJV. The IJV (V) is superficial to the carotid artery (A). The guide wire can be seen as a bright, hyperechoic line (G) crossing the wall of the vein and then remaining in the lumen of the jugular vein.

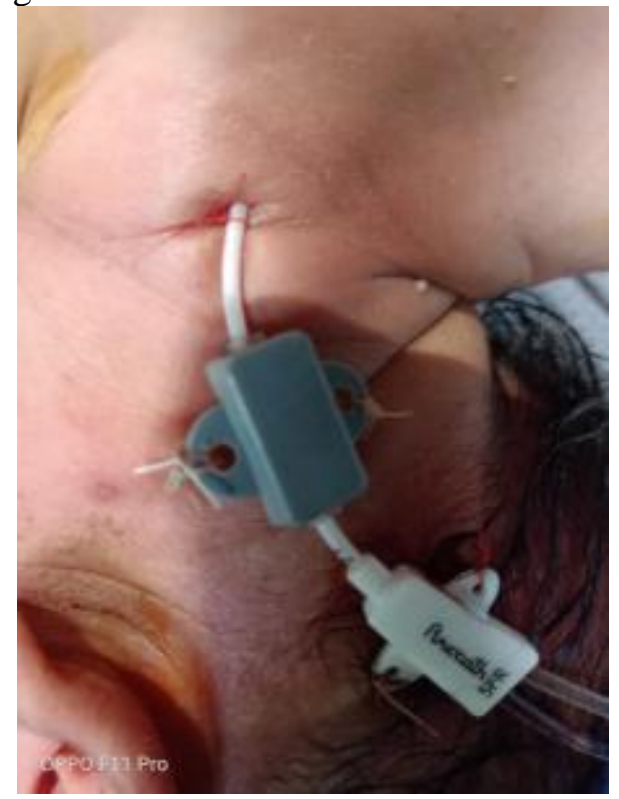

Fig. (7)

b- Subclavian vein catheterization: The child was positioned in the Trendelenburg position with a towel roll running cranial to caudal under the thoracic spine to support the sternum vertically above the level of the shoulders. The needle was inserted just lateral to the proximal angle of the clavicle, where the medial third and lateral twothirds of the clavicle meet. The needle was inserted under the distal third of the clavicle, slightly cephalad toward the sternal notch. The path of the needle was passed under the clavicle. When blood flow was obtained, the Seldinger technique principles were followed. Three attempts were performed, and if failed another vessel was accessed.

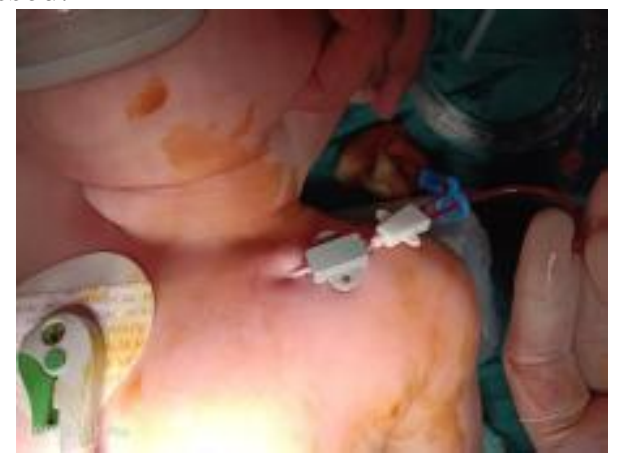

Fig. (8)

Evaluation and follow up: Dressing was applied in all cases. A three-way adaptor was also connected to the intravenous catheter. The position of catheter was confirmed by a chest X-ray, which should be in the superior vena cava, outside the cardiac reflection and above the T2 vertebra. Daily catheter inspection was done for occlusion and leakage and evaluation and follow up of vital signs especially temperature, signs of local CVC inflammation (hotness, tenderness, redness and swelling), signs of local CVC infection (the presence of discharge, pus, enlarged lymph node), developing of endocarditis.

\section{Following data were recorded for all patients in both groups:}

- Demographic data: Included patient age, sex, weight, height, previous central venous catheterization, venous thromboses, and preoperative investigations for great vein patency.

- CVC data: Included type, external diameter, indication for insertion and whether or not it was a reinsertion, intended use, the requesting department, and the vein into which the CVC was inserted.

- Catheterization success rate: Defined as ability to cannulate the vein in less than three trials (Inability to cannulate the vein in three attempts was recorded as a failure) in ultrasound guided group. In cut-down group it was defined as ability to visualize, located and cannulate the vein. (Improper plane, accidental penetration of carotid artery was recorded as a failure)

- Number of attempts: For insertion of CVC.

- Venous access time: Defined as the time from the starting of the procedure to the return of dark colored venous blood into the attached syringe, not including the time of suturing, fixation of catheter and dressing.

- Catheterization time: Defined as the time from the starting of procedure to the end of catheter placement, including the suturing and fixation time.

- Technical feasibility: Defined as clinical experience with the steps of the procedure of CVC represented by easy or difficult technique and percentage in each group.

- Complications: Failure to cannulate any vein. Hematoma at site of insertion. Malposition of the catheter. Right atrial perforation. Arterial puncture. Cardiac arrhythmias. Pneumothorax. Hemothorax.

- Post-procedure chest x-ray: Immediately after insertion for all patients to confirm the position of the catheter tip and early detection of complications. For intraoperative CVC chest X-ray was done immediately at the end of surgery. Complications, if occurred were managed according to the standard protocol. 


\section{Statistical analysis}

Recorded data were analyzed using the statistical package for social sciences, version 20.0 (SPSS Inc., Chicago, Illinois, USA). Quantitative data were expressed as mean \pm standard deviation (SD). Qualitative data were expressed as frequency and percentage.

The following tests were done: Independent-samples t-test of significance was used when comparing between two means. Chi- square $\left(\mathrm{x}^{2}\right)$ or Fisher's exact test of significance was used in order to compare proportions between qualitative parameters. The confidence interval was set to $95 \%$ and the margin of error accepted was set to $5 \%$. So, the $\mathrm{p}$-value was considered significant as the following: $\mathrm{P}$-value $<0.05$ was considered significant. P-value $<0.001$ was considered as highly significant. P-value $>0.05$ was considered insignificant.

\section{RESULTS}

Table (1): Comparison between group A and group B according to demographic data.

\begin{tabular}{|l|c|c|c|c|}
\hline Parameter & $\begin{array}{c}\text { Group A } \\
\mathbf{N}=\mathbf{5 0}\end{array}$ & $\begin{array}{c}\text { Group B } \\
\text { No=50 }\end{array}$ & t-test & P-value \\
\cline { 1 - 3 } Age (months) & $7.67 \pm 2.15$ & $7.52 \pm 2.10$ & 0.353 & 0.725 \\
\hline Sex & & & & \\
\cline { 1 - 3 } Male & $28(56.0 \%)$ & $30(60.0 \%)$ & \multirow{2}{*}{0.041} & 0.839 \\
\cline { 1 - 3 } Weight (kg) & $22(44.0 \%)$ & $20(40.0 \%)$ & & 0.717 \\
\hline Height (cm) & $9.44 \pm 2.64$ & $9.25 \pm 2.59$ & 0.363 & 0.468 \\
\hline
\end{tabular}

This table shows no statistically significant difference between group A and group B according to demographic data.

Table (2): Comparison between group A and group B according to site of insertion.

\begin{tabular}{|l|c|c|}
\hline Site of insertion & $\begin{array}{c}\text { Group A } \\
\mathbf{N = 5 0}\end{array}$ & $\begin{array}{c}\text { Group B } \\
\text { No=50 }\end{array}$ \\
\hline Right internal jugular vein & $45(90.0 \%)$ & $25(50.0 \%)$ \\
\hline Left internal jugular vein & $5(10.0 \%)$ & $20(40.0 \%)$ \\
\hline Right subclavian vein & $0(0.0 \%)$ & $5(10.0 \%)$ \\
\hline
\end{tabular}

This table shows the frequency of the different sites of insertion in the 2 groups.

Table (3): Comparison between group A and group B according to induction for insertion.

\begin{tabular}{|l|c|c|c|c|}
\hline Indication for insertion & $\begin{array}{c}\text { Group A } \\
\text { N=50 }\end{array}$ & $\begin{array}{c}\text { Group B } \\
\text { No=50 }\end{array}$ & x2 & $\begin{array}{c}\text { p- } \\
\text { value }\end{array}$ \\
\cline { 1 - 3 } Needs parenteral nutrition & $27(54.0 \%)$ & $30(60.0 \%)$ & \multirow{2}{*}{0.431} & \multirow{2}{*}{0.806} \\
\cline { 1 - 3 } Needs for inotropic drugs & $18(36.0 \%)$ & $15(30.0 \%)$ & \\
\cline { 1 - 3 } Needs for exchange blood transfusion & $5(10.0 \%)$ & $5(10.0 \%)$ & & \\
\cline { 1 - 3 }
\end{tabular}

This table shows no statistically significant difference between group A and group B according to indication for insertion.

Table (4): Comparison between group A and group B according to success rate.

\begin{tabular}{|l|c|c|c|c|}
\hline Success rate & $\begin{array}{c}\text { Group A } \\
\mathbf{N = 5 0}\end{array}$ & $\begin{array}{c}\text { Group B } \\
\text { No=50 }\end{array}$ & x2 & p-value \\
\hline Successful & $50(100 \%)$ & $45(90 \%)$ & \multirow{2}{*}{3.368} & 0.067 \\
\hline Failed & $0(0 \%)$ & $5(10 \%)$ & \\
\hline
\end{tabular}

This table shows no statistically significant difference between group A and group B according to success rate.

Table (5): Comparison between group A and group B according to technical feasibility.

\begin{tabular}{|l|c|c|}
\hline Technical feasibility & $\begin{array}{c}\text { Group A } \\
\text { N=50 }\end{array}$ & $\begin{array}{c}\text { Group B } \\
\text { No=50 }\end{array}$ \\
\hline Tunneled & $10(20 \%)$ & $0(0 \%)$ \\
\hline Non tunneled & $40(80 \%)$ & $0(0 \%)$ \\
\hline Ultrasound guided & $0(0 \%)$ & $50(100 \%)$ \\
\hline
\end{tabular}

This table shows the frequency of different techniques that were used in this study. 
Table (6): Comparison between group A and group B according to anesthesia.

\begin{tabular}{|l|c|c|c|c|}
\hline Anesthesia & $\begin{array}{c}\text { Group A } \\
\text { N=50 }\end{array}$ & $\begin{array}{c}\text { Group B } \\
\text { No=50 }\end{array}$ & x2 & p-value \\
\hline Local anesthesia & $43(86.0 \%)$ & $47(94.0 \%)$ & \multirow{2}{*}{1.000} & 0.317 \\
\hline General anesthesia & $7(14.0 \%)$ & $3(6.0 \%)$ & \\
\hline
\end{tabular}

This table shows no statistically significant difference between group A and group B according to anesthesia.

Table (7): Comparison between group A and group B according to number of attempts and technical feasibility.

\begin{tabular}{|c|c|c|c|c|}
\hline Parameter & $\begin{array}{c}\text { Group A } \\
\mathbf{N}=50\end{array}$ & $\begin{array}{c}\text { Group B } \\
\text { No }=50\end{array}$ & $x^{2}$ & p-value \\
\hline \multicolumn{5}{|c|}{ No of attempts } \\
\hline 1 & $50(100.0 \%)$ & $47(94.0 \%)$ & \multirow{3}{*}{1.375} & \multirow{3}{*}{0.241} \\
\hline 2 & $0(0.0 \%)$ & $1(2.0 \%)$ & & \\
\hline 3 & $0(0.0 \%)$ & $2(4.0 \%)$ & & \\
\hline \multicolumn{5}{|c|}{ Technical feasibility } \\
\hline Easy & $49(98.0 \%)$ & $46(92.0 \%)$ & \multirow{2}{*}{0.842} & \multirow{2}{*}{0.359} \\
\hline Difficult & $1(2.0 \%)$ & $4(8.0 \%)$ & & \\
\hline
\end{tabular}

This table shows no statistically significant difference between group A and group B according to number of attempts and technical feasibility.

Table (8): Comparison between group A and group B according to complications.

\begin{tabular}{|l|c|c|c|c|}
\hline Complications & $\begin{array}{c}\text { Group A } \\
\text { N=50 }\end{array}$ & $\begin{array}{c}\text { Group B } \\
\text { No=50 }\end{array}$ & $\mathbf{x}^{\mathbf{2}}$ & p-value \\
\hline Hematoma & $1(2.0 \%)$ & $2(4.0 \%)$ & 0.074 & 0.786 \\
\hline Malposition & $1(2.0 \%)$ & $2(4.0 \%)$ & 0.074 & 0.786 \\
\hline Arterial puncture & $0(0.0 \%)$ & $2(4.0 \%)$ & 0.510 & 0.475 \\
\hline Cardiac arrhythmia & $1(2.0 \%)$ & $1(2.0 \%)$ & 0.000 & 1.000 \\
\hline Pneumothorax & $0(0.0 \%)$ & $2(4.0 \%)$ & 0.510 & 0.475 \\
\hline Hemothorax & $0(0.0 \%)$ & $2(4.0 \%)$ & 0.510 & 0.475 \\
\hline Occlusion & $4(8.0 \%)$ & $5(10.0 \%)$ & 0.074 & 0.786 \\
\hline accidental dislodgement & $5(10.0 \%)$ & $3(6.0 \%)$ & 0.510 & 0.475 \\
\hline Infections due to & $5(10.0 \%)$ & $3(6.0 \%)$ & 0.510 & 0.475 \\
\hline $\begin{array}{l}\text { Catheter removed } \\
\text { complications }\end{array}$ & $4(8.0 \%)$ & $3(6.0 \%)$ & 0.074 & 0.786 \\
\hline Culture of catheter tip (No-growth) & $15(30.0 \%)$ & $15(30.0 \%)$ & 0.000 & 1.000 \\
\hline
\end{tabular}

This table shows no statistically significant difference between group A and group B according to complications.

Table (9): Comparison between group A and group B according to culture of catheter tip.

\begin{tabular}{|l|c|c|c|c|}
\hline Culture of catheter tip & $\begin{array}{c}\text { Group A } \\
\text { N=50 }\end{array}$ & $\begin{array}{c}\text { Group B } \\
\text { No=50 }\end{array}$ & \multirow{2}{*}{$\mathbf{X}^{\mathbf{2}}$} & p-value \\
\cline { 1 - 3 } No growth & $15(30.0 \%)$ & $15(30.0 \%)$ & \multirow{2}{*}{0.033} & \multirow{2}{*}{0.855} \\
\hline Positive for gram +ve cocci & $5(10.0 \%)$ & $6(12.0 \%)$ & \\
\hline
\end{tabular}

This table shows no statistically significant difference between group A and group B according to culture of catheter tip.

\section{DISCUSSION}


Legler and Nugent (7) described ultrasound as an assisting device for central venous catheterization. Ultrasound guidance can be provided either through the external application of ultrasound probe to visualize and locate the central veins or by using Doppler probe for identifying needle entry into the vein. National Institute of Clinical Excellence (NICE) guidelines in $2002^{(8)}$, recommend ultrasound guidance when central venous catheterization is needed.

Many literatures comparing the percutaneous anatomical landmark technique with the ultrasound-guided technique for $\mathrm{CVC}$ in children. However, studies comparing the surgical cut down technique with ultrasound-guided technique CVC in children are lacking. This present study compare the surgical cut down technique with ultrasound-guided technique CVC in 100 of infants and neonates.

In the present study, The CVC under ultrasound guidance were successfully achieved in 50 patients of group B (100\% of patients). On the other hand, the surgical cut down technique using anatomical landmarks was used in 50 patients in group A and also had a successful CVC in $100 \%$ of patients. The reported difference was related to number of attempts during CVC, venous access time, catheterization time, technical feasibility and complications.

Mallory et al. ${ }^{(9)}$ and Denys et al. ${ }^{(10)}$ found a significant difference in success rate while comparing the percutaneous anatomical landmark technique with the ultrasound-guided technique. Also, Chuan et al. ${ }^{(11)}$ found statistically significant difference in success rate between the percutaneous anatomical landmark technique and the ultrasound-guided technique ( $80 \%$ vs. $100 \%$ ) in their study, in infants. Also they reported significant difference in median venous access time and the median catheterization time. Early in the course of this study there was significant difference in median venous access time and the median catheterization time between cut down technique and ultrasound guided technique. Later, after learning curve with ultrasound guided technique, this difference became insignificant.

In group $\mathbf{A}$ of patients the cut-down technique found success at the first attempt in all patients (100\%). Incidence of arterial puncture was not reported in this group of patients due to direct visualization of the vein. In group B of patient's ultrasound-guided technique, found success at the first attempt in 45 patients (90\%).

Asheim et al. ${ }^{(12)}$ performed cannulation of the central vein guided by an ultrasound technique. They were successful in all 42 infants and children. They found that the vein was punctured during the first attempt in 40 out of 42 infants undergoing CVCs guided by ultrasound.

Malbezin et al. ${ }^{(13)}$ reported in the cohort of 5434 patients, on percutaneous pediatric central venous catheters targeted by experienced and specifically trained operators using landmark technique successful cannulation occurred in 99.5\% with a perioperative complication rate of $1.3 \%$. They demonstrated that global complication rates are not necessarily comparable across all studies as no standard complications have been defined for CVC procedure, and the inclusion of lesser complications varies from study to study. Other studies by Araujo et al. ${ }^{(14)}$ reported $10.8 \%$ failure rate by using landmark technique. Grebenik et al. ${ }^{(15)}$ also reported the same failure rate during comparison of ultrasound-guided and landmark technique. Both these studies were performed on smaller children. Mean weights in these studies were 5.8 and $9 \mathrm{~kg}$ respectively, compared with $19 \mathrm{~kg}$ in Malbezin et al. ${ }^{(13)}$ study. Furthermore, Araujo et al. ${ }^{(14)}$ did not employ general anesthesia and involved emergent conditions.

In the present study no failure rate was reported in group A (cut down group). However, $4 \%$ failure rate was reported in group B (ultrasound guided group) and occur in two cases of 50 cases of the group, and the explanation could be due to small low birth weight of the first case infant and second one present with generalized edema, moreover at that time we were at the beginning of our learning curve, and both cases converted to surgical cut down technique.

Alderson et al. ${ }^{(16)}$ surprisingly reported that $18 \%$ of children had anomalous venous anatomy and that ultrasound guided CVC reduces both the time and number of needle insertions required to aspirate venous blood. However, Asheim et al. (12) on they meta-analysis on ultrasound guided pediatric CVC insertions did not show any reduction in complications or failure rate when used even by skilled operators. Malbezin $\boldsymbol{e t}$ al. ${ }^{(13)}$ reported that children's weight under $3 \mathrm{~kg}$ was predictive of failure, as smaller veins are more difficult to cannulate. Similarly, hemodialysis CVC was another predictive of failure may be explained by their large diameters in comparison with patients' veins. The data suggested a learning curve.

This present study results confirm the assumption that specifically trained skilled operators should expect low failure rates when using ultrasound guided CVC in children.

Arul et al. ${ }^{(17)}$ reported $2.4 \%$ complication rate in a 500 patient pediatric using ultrasound guided CVC, and Janik et al. ${ }^{(\mathbf{1 8})}$, reported $7.9 \%$ in 
children and infants; where much larger diameter CVCs were used. It is noteworthy that arterial punctures comprised $25 \%$ of all complications in most studies. Bleeding disorders were corrected before CVC insertions, and simple arterial puncture without dilation and wire passage was not recorded, also, the more serious events of intraarterial wire passage and dilation (CVC position) were recorded in this present study.

Malbezin et al. ${ }^{(13)}$ in two cases, dilation of an artery occurred - one recognized during the procedure and one by CVC position on postoperative X-ray

Malbezin et al. ${ }^{(13)}$ reported hemothorax and pneumothorax rates of $0.1 \%$. Arul et al. ${ }^{(17)}$ reported no hemothoraces or pneumothoraces in 500 internal jugular CVC. In most literatures, pneumothorax and hemothorax rates are generally reported around $1-2 \%{ }^{(4,5)}$. Internal jugular vein CVC was targeted preferentially following reports of complications in subclavian CVC insertions in children. Moreover, high risk of thrombosis of subclavian veins in children were reported ${ }^{(13)}$. Therefore, it seems reasonable to target extrathoracic veins where the safety margin is higher.

In a large series of $5434 \mathrm{CVC}$ in children, they reported that central venous insertions may be contributed to catastrophic event of death in two cases, occurred within 30 days' post procedure. They reported that the first baby was suffering from multiple organ failure and died from cardiorespiratory failure with hemothorax 48 hour after CVC. The second was born with congenital atrioventricular septal defect and intestinal atresia, and submitted to bowel resection with tunneled CVC on the first day of life. The CVC was removed on day 12 due to obvious tunnel infection. Overwhelming staphylococcal sepsis ensued, and the patient died on day 13 .

In this present study, no reported cases of deaths. CVC malposition rates were reported in children as $1.2 \%$ by Arul et al. ${ }^{(17)}, 7.3 \%$ by Janik et al. ${ }^{(18)}$, and $0.6 \%$ by Malbezin et al. ${ }^{(13)}$. There is a debate about the routine use of fluoroscopic guidance after CVC in children. Some authors recommend operative fluoroscopic guidance when long-term CVC is considered. On the other hand, many authors reliably estimated CVC insertion depths without fluoroscopic guidance. Despite this debate for the use of intraoperative fluoroscopy, the standard postoperative chest $\mathrm{X}$-ray is recommended in most literatures, and its routine post-insertion use appears justified ${ }^{(\mathbf{( 1 8})}$.

In the present study, the optimal length of insertion of CVC was adjusted according to the following protocol; the correct length of insertion $(\mathrm{cm})=($ height in $\mathrm{cm} / 10)-1$ for children less than or equal $100 \mathrm{~cm}$ in height, and (height in $\mathrm{cm} / 10$ )-2 for children more than $100 \mathrm{~cm}$ in height as described by Kayashima et al. ${ }^{\left({ }^{19)}\right.}$ and all cases of CVC in both groups of patients (surgical cut-down and ultrasonic guided insertion) submitted to routine post-insertion chest $\mathrm{x}$-ray to estimate CVC insertion depths and to detect any complications like hemothoraces or pneumothoraces.

Regarding Central venous catheter size and its relation to child age, height, or weight, no clear recommendations exist in the literatures. Some literatures described a relationship between internal jugular vein diameter and height, weight, age, and body surface area in children. However, practically the diameter of the internal jugular vein was poorly predicted by patient age. However, it is demonstrated that, the risk of catheter complications increased when using $6 \mathrm{Fr} / 2 \mathrm{~mm}$ CVCs in patients below 1 year of age. Malbezin $\boldsymbol{e t}$ al. ${ }^{(13)}$ demonstrated a protocol for central venous catheter diameters according to child weight, and they reported that this protocol is empirical, but they used it for two decades with acceptable results.

In the present study, the central venous catheter size in both groups of this study was chosen according to patient weight, by the using the following protocol children below $3 \mathrm{~kg}$ : 3 French catheter size, children from 3-10 kg: 4 Fr catheter size, children from 10-25 kg: $5 \mathrm{Fr}$ catheter size. The same protocol as described by Malbezin et $\boldsymbol{a l} .{ }^{(13)}$ and practically we, found it very easy with less complications and that the relation between catheter size and vein size was appropriate in most cases.

\section{CONCLUSION}

The present study reported that the ultrasound guided CVC was found to be more useful in placement of CVC in infants and neonates, and decreasing complications. The ultrasound technique not only clarifies the relative position of the vein and its surrounding structures but also helps in identifying the course of the central vein and its caliber and provide confidence to the operator. Cost is one of the limiting factors in the availability of ultrasound device in many clinical applications. Ultrasound machine available in operation room or ICUs for other purposes can be used for ultrasound-guided CVC, thereby increasing successful cannulation, effective utilization of available equipment and avoiding the purchase of additional equipment. 


\section{REFERENCES}

1. Ares G, Hunter CJ (2017): Central venous access in children: indications, devices, and risks. Curr Opin Pediatr., 29:340-346.

2. Forssmann W (1929): Die sondierung des rechten herzens. Journal of Molecular Medicine, 8(45):2085-7.

3. Aubaniac R (1952): Subclavian intravenous injection; advantages and technic. Presse Med., 60:1456-59.

4. Lin KY, Cheng A, Chang YC et al. (2017): Central line-associated bloodstream infections among critically-ill patients in the era of bundle care. J Microbiol Immunol Infect., 50:339-48.

5. Bhutta A, Gilliam C, Honeycutt M et al. (2007): Reduction of bloodstream infections associated with catheters in pediatric intensive care unit: stepwise approach. BMJ., 334:362-5.

6. Ge X, Cavallazzi R, Li C et al. (2012): Central venous access sites for the prevention of venous thrombosis, stenosis and infection. doi:10.1002/14651858.CD004084.pub3 . PMC 6516884. PMID 22419292

7. Legler D, Nugent M (1984): Doppler localization of the internal vein facilitates central venous cannulation. Anesthesiology, 60:481-6.

8. National Institute for Clinical Excellence (2002): Guidance on the Use of Ultrasound Locating Devices for Placing Central Venous Catheters. NICE Technology Appraisal Guidance , https://www.nice.org.uk > guidance > resources

9. Mallory DL, McGee WT, Shawker TH et al. (1990): Ultrasound guidance improves the success rate of internal jugular vein cannulation. Chest, 98:157-60.

10. Denys BG, Uretsky BF, Reddy PS (1993): Ultrasound- assisted cannulation of the internal jugular vein. A prospective comparison to the external landmark guided technique. Circulation, 87:1557-62.
11. Chuan WX, Wei W, Yu L (2005): A randomized- controlled study of ultrasound prelocation versus anatomical landmark guided cannulation of the internal jugular vein in infants and children. Paediatr Anaesth., 15:733-8.

12. Asheim P, Mostad U, Aadahl P (2002): Ultrasound-guided central venous cannulation in infants and children. Acta Anaesthesiol Scand., 46:390-2.

13. Malbezin S, Gauss T, Smith I et al. (2013): A review of 5434 percutaneous pediatric central venous catheters inserted by anesthesiologists. https://www.researchgate.net/publication/2366736 68_A_review_of_5434_percutaneous_pediatric_ce ntral_venous_catheters_inserted_by_anesthesiolog ists

14. Araujo CC, Lima MC, Falbo GH et al. (2007): Percuta-neous subclavian central venous catheterization in children and adolescents: success, complications and related factors. J Pediatr(Rio J), 83:64-70.

15. Grebenik CR, Boyce A, Sinclair ME et al. (2004): NICE guidelines for central venous catheterization in children. Is the evidence base suffi-cient? Br J Anaesth., 92: 827-830.

16. Alderson PJ, Burrows FA, Stemp LI et al. (1993): Use of ultrasound to evaluate internal jugular vein anatomy and to facilitate centralvenous cannulation in paediatric patients. Br J Anaesth., 70: $145-148$.

17. Arul GS, Lewis N, Bromley P et al. (2009): Ultrasound-guided percutaneous insertion of Hickman lines in children. Prospective studyof 500 consecutive procedures. J Pediatr Surg., 44: 13711376.

18. Janik JE, Conlon SJ, Janik JS (2004): Percutaneouscentral access in patients younger than5 years: size does matter. J Pediatr Surg., 39: 1252-1256.

19. Kayashima K, Uezonoj S, Andropoulos DB (2015): Anesthesia for congenital heart disease. 3rd Edition. Wiley-Blackwell. 